\title{
Leader Group Prototypicality and Employee Well-Being: The Mediate Effect of Group Commitment and the Moderate Effect of Openness
}

\author{
Xinxing Guo \\ Jinan University, Guangzhou, China \\ Email: 737845483@qq.com
}

Received 31 March 2016; accepted 23 April 2016; published 26 April 2016

Copyright (C) 2016 by author and Scientific Research Publishing Inc.

This work is licensed under the Creative Commons Attribution International License (CC BY). http://creativecommons.org/licenses/by/4.0/

\begin{abstract}
According to the social identity Theory, leadership group prototypicality have an important effect in employee's group identity, work attitude and employee well-being. In this article, we explore the mechanism between leadership group prototypicality and employee well-being to understand how leadership group prototypicality works. We had a sample of 336 employees to test our hypothesis in communication carriers in Guangdong. We used the longitudinal survey and collected the data in three different time point. At last, regression method was used to analyze the data. The results showed that: 1) leadership group prototypicality group could predict employee well-being; 2) mediating effect of group commitment was proud between leadership group prototypicality and employee well-being; 3) moderating effects of openness were found upon the relationship between leadership group prototypicality and group commitment. Finally, this study proposed management practice for leadership group prototypicality, and proposed future research prospects.
\end{abstract}

\section{Keywords}

Leader Group Prototypicality, Employee Well-Being, Group Commitment, Openness

\section{Introduction}

More and more researchers have been attention to leadership to improve the effectiveness of the leadership, and the level of employees’ happiness (Ullrich, Christ, \& Dick, 2009; Dijke \& Cremer, 2008, 2010). As with work environment and employees changing quickly, how to improve the effectiveness of management by increasing 
employee trust and investment has been an urgent problem to be solved. In a team, if employee can agree with leader's value, behavior characteristic and decision making style, they may view what leaders say and do as their own views, then become more recognized with organization and leadership (Basford, Offermann \& Behrend, 2014), thereby receive happiness.

Hogg (2001) proposes that leader group prototypicality means leaders have common property of the team he belongs to. Some research demonstrates that leader group prototypicality has a positive impact on cognitive and affective of employees. Employee well-being refers to the employee and has positive state of mind in their work, so we hypothesize that the leader group prototypicality has a positive impact on the well-being of employees. There are empirical studies that have shown that team with a high prototypicality of leader may have a higher positive emotions (Kuoppala et al., 2008; Skakon et al., 2010). Olkkonen (2005) proves that with the higher leader group prototypicality, staff is more intense to fairness, and intends to perform higher self-evaluation and pride. In addition, the researchers note that the staff of the organization identity can predict employee well-being, which means organizational identity can make employee experience more happiness (Wegge et al., 2008).

According to social identity theory (Tajfel, 1978), people always look for external tags to complete self-identity. From the perspective of team commitment, the sense of identity leads to positive perception of the community commitment and emotional commitment, and a strong sense of happiness (Alexandra \& Christian, 2008). Leader group prototypicality is not only influenced by employees' emotional and cognitive, but also influenced by employees' personality traits. The researcher finds that employee with high openness can be satisfied easily (Starbuck, 2015). This also shows that openness can strengthen the relationship between leaders and employees.

Above all, we can consider that leader group prototypicality closely relates to the well-being of employees. But the mechanism of how leader group prototypicality influence on employee well-being is not clear. Therefore, in this study, we examine the relationship between leader group prototypicality and employee well-being, and the moderating effects of openness personality on this relationship.

\section{Theoretical Background and Hypotheses}

\subsection{Leader Group Prototypicality, Group Commitment and Employee Well-Being}

Leader Group Prototypicality and Employee Well-being. Employee well-being is subjective understanding and evaluation by employee in the work environment and the subjective well-being in the areas of work mainly reflect on job satisfaction. Leader group prototypicality can influence employees' perceptions and attitudes to obtain recognition of subordinates. Because of the recognition, employees will be considered decision made by leader represent their own opinions, thereby have a sense of satisfaction. Empirical studies also showed that the subordinates work for leader who with a high group prototypicality job satisfaction is higher (Cicero, Pierro, \& van Knippenberg, 2007). Employee well-being and obtained enhance in work has a positive correlation (Ryff \& Dinger, 2006). Ryff (2006) pointed out that self-acceptance, positive relations with others, autonomy, environmental controllability, life goals and personal growth can improve well-being of employees. Leaders have high group prototypicality actively focus on employee opinions and attitudes, and help employees achieve self-identity (Giessner \& van Knippenberg, 2008). Moreover, positive and rich psychological resources make employees feel happiness. Employees need deal with the pressure from work, and a high prototypicality of leader can provide staff support and assistance (Bakker \& Demerouti, 2007), so that they have adequate resources to face the pressure of work, then promote the well-being.

Leader Group Prototypicality and Group Commitment. Based on social identity theory, individuals' attitude, behavior and behavioral tendencies tend to consistency with others (Jimmieson et al., 2008). Leader group prototypicality means the team leader' traits and team' values are close, so the group leader with a high prototypicality represent the core characteristics of this team (Knippenberg, 2011). Team members can use those qualities to distinguish in-group and out-group members, and during the course of distinction, the team number may form concept of "we", to get the sense of belonging (Michae, van Knippenberg \& David, 2012). This means that if leader group prototypicality is high, the team members are more likely to believe their leader is part of the team, their level of trust will become higher, which makes team members feel like in a "community". On the other hand, when employees perceive a higher prototypicality of the leader, they may think the team is worthy to trust. Thus, the relationship between members and leader become closer for trust and lead to a higher sense of commitments. Studies have shown that leader group prototypicality can promote cooperation willingness of employees (De Cremer, van Dijke, \& Mayer, 2010), which can coordinate the relationship between team 
members, and have a high group commitment.

To sum up, we hypothesize:

Hypothesis 1: Leader group prototypicality will have a significant and positive impact on employee wellbeing.

Hypothesize 2: Leader group prototypicality has a significant and positive impact on group commitment.

\subsection{Group Commitment as a Mediator of Leader Group Prototypicality and Employee Well-Being}

It has often been claimed that group commitment have a certain influence on employee well-being. Ajay (2013) proved the group commitment have a positive role in promoting employee well-being. In a recent study by Alexandre et al. (2015) that the employee with high group commitment has a higher sense of belonging, responsibility and ownership, and their levels of subjective well-being are higher too. To sum up, the higher the team commitment employees is, the more positive emotions can be experienced, and employee will be happier. According to social identity theory, leader with high group prototypicality may get more support when make a decision. As commitment is core elements of positive psychology, which could promote the identity of leader, thereby improve the well-being of employees (David et al., 2014). According to the theory of self-determination, employees have a demand of self-management. When employees feel their works are in control, they will show higher organizational commitment, and experience the happiness (Slemp \& Vella-Brodrick, 2014). Based on the analysis, leader group prototypicality having a positive relationship with team commitment, and team commitment can improve employee well-being. Thus we proposed that leader group prototypicality influence the well-being of employees through team commitment.

Hypothesize 3: Group commitment positively mediates the positive relationship between leader group prototypicality and employee well-being.

\subsection{Openness as a Moderator of Leader Group Prototypicality and Group Commitment}

Employees with high openness are desire to explore relationships (Piedmont, 1998), Which led to a higher degree of acceptance of others, and willing to understand others who are different, so people with high openness are more easier generate interpersonal links ,such as commitment (Zhao, Seibert, \& Lumpkin, 2010). These resources not only materially, but also on the work and interpersonal. So employees who with high openness may easier form group commitment under the influence of the leadership. From another perspective, employees always face uncertainty challenges in a work environment. Most of employees want to reduce uncertainty (Hogg, 2000; Hogg, 2007), and it will consume psychological resources. Pierro et al. (2005) conducted a series of study point out that in order to reduce the uncertainty, people may close their cognition, such as avoid judgment, decisions and actions to avoid risks. Choi, Oh, \& Colbert (2015) found that employee with high level of happiness may have high level of group commitment too.

Employees can reduce uncertainty through the relationship between the share of social reality, social identity. In other words, they reduce employee uncertainty dependent on groups and leadership. Furthermore, Employees with open personality of risk have less perception of uncertainty, and is easier to accept the leader's influence, thus form group commitment. Accordingly, we hypothesize:

Hypothesize 4: Openness moderates the indirect relationship between leader group prototypicality and group commitment, in that the indirect relationship will become stronger when openness is stronger.

\section{Method}

\subsection{Participants and Procedure}

We collected data from several companies located in south of China, including private enterprises, nationalized business, foreign-owned enterprises, joint ventures, and public institutions. Data were collected by conducting two-wave longitudinal survey for six months. The Wave 1 surveyed the variable of 6 months and organizational support. Six months later, the Wave 2 surveyed the variable of psychology safety and change-supportive behavior. We randomly distributed 397 questionnaires, finally received 366 valid questionnaires for a response rate of $92.19 \%$. Of the respondents, $29.4 \%$ were males and $70.6 \%$ were females, and average age was 29.83 years. 
The type of work distribution was engineering (29.1\%), administration (19\%), marketing (20.2\%) and manufacture (15.8\%), respectively.

\subsection{Measures}

The measures we used in this study have been verified previously with good reliability and validity in the Chinese culture and in other countries and cultures. In this study, all scale items were rated on a 7-point Likert scale ranging from $1=$ strongly disagree to $7=$ strongly agree.

Leader Group Prototypicality. To measure leader group prototypicality, we use van Knippenberg's (2005) six-item scale. One item is "My team leader can represent the characteristics of the members of our team". The coefficient alpha was 0.902 , respectively, providing evidence that the measure was highly reliable.

Openness. We measured openness using the Mini-Marker Big-Five Scale revised by Saucier (1994), include eight-item which are adjective. We asked subjects evaluate how much each of the adjectives can describe their own character. Cronbach's alpha for the scale was 0.763 , providing evidence that the measure was highly reliable.

Group Commitment. Based on Bishop and Scott (2000), we used eight items to measure group commitment. In this study, the group commitment measures the subjects' acceptance of team goals, values and willing to make efforts for team. Cronbach's alpha for the scale was 0.821 , providing evidence that the measure was highly reliable.

Employee Well-being. We use the Oxford Happiness Questionnaire to measure employee well-being, which developed by Hills and Argyle (2000). The scale which has eight items, is a measure of subjective well-being of individuals. Cronbach's alpha for the scale was 0.867 , providing evidence that the measure was highly reliable.

\section{Data Analysis and Results}

\subsection{Description and Correlation Analysis}

The means and standard deviations of the major variables and the correlation coefficients among the variables are shown in Table 1. Correlation analysis showed that leader group prototypicality had a significant and positive correlation with openness, group commitment and employee well-being ( $\mathrm{r}=0.21, p<0.01 ; \mathrm{r}=0.41, p<$ $0.01 ; \mathrm{r}=0.29, p<0.01$ ); group commitment had a significant and positive correlation with employee well-being $(\mathrm{r}=0.42, p<0.01)$. According to Tsui et al. (1995) findings, there is a serious multicollinearity problem when the related levels are higher than the standard value of 0.75 . However, the above analysis showed that there was no existing multicollinearity problem and that it was suitable for further regression analysis.

\subsection{Leader Group Prototypicality, Group Commitment and Employee Well-Being}

We ran mediation bias-corrected bootstrapping resampling method with 5000 resamples (by means of PROCESS; Preacher \& Hayes, 2008; updated by Hayes, 2013) to examine whether the impact leader group prototypicality on employee well-being was mediated by group commitment. As can be seen in Table 2, analysis revealed that leader group prototypicality have a significant effect on employee well-being $(B=0.142, p<0.000)$, supporting Hypothesis 1; leader group prototypicality have a significant effect on group commitment $(B=0.250, p<0.05)$, supporting Hypothesis 2 . When mediating variables is not been controlled, leader group prototypicality have a significant effect on employee well-being $(B=0.142, p<0.000)$, and mediating variables has been controlled,

Table 1. Mean, standard deviations, and correlation.

\begin{tabular}{lcccccccc}
\hline \multicolumn{1}{c}{ Variate } & Mean & s.d. & $\mathbf{1}$ & $\mathbf{2}$ & $\mathbf{3}$ & $\mathbf{4}$ & $\mathbf{5}$ & $\mathbf{6}$ \\
\hline 1 Gender & - & - & & & & & & \\
2 Age & 29.83 & 4.22 & 0.00 & & & & \\
3 Educational background & 5.32 & 0.66 & -0.09 & -0.07 & & & \\
4 Leader group prototypicality & 5.07 & 1.15 & 0.06 & 0.01 & 0.02 & & & \\
5 Openness & 4.97 & 0.86 & $-0.15^{* *}$ & 0.06 & $0.15^{* *}$ & $\mathbf{0 . 2 1}^{* *}$ & & \\
6 Group commitment & 5.43 & 1.08 & 0.05 & -0.02 & 0.08 & $\mathbf{0 . 4 1}^{* *}$ & $\mathbf{0 . 2 9}^{* *}$ & \\
7 Employee well-being behavior & 4.71 & 0.93 & -0.01 & -0.03 & $0.15^{* *}$ & $\mathbf{0 . 2 9}^{* *}$ & $\mathbf{0 . 3 4}^{* *}$ & $\mathbf{0 . 4 2}^{* *}$ \\
\hline
\end{tabular}

Note1: ${ }^{* * *} p<0.001 ;{ }^{* *} p<0.01 ;{ }^{*} p<0.05$. 
Table 2. Regression analysis result for the hypothesized relationships $(\mathrm{N}=366)$.

\begin{tabular}{lcccc}
\hline \multicolumn{1}{c}{ Variables } & coeff & SE & t & P \\
\hline Independent variable to mediating variables (Path a) & 0.250 & 0.040 & 2.617 & 0.000 \\
Mediating variables to dependent variable (Path b) & 0.087 & 0.048 & 4.139 & 0.000 \\
Independent variable to dependent variable (Path C, not control mediating variables ) & 0.142 & 0.040 & 3.530 & 0.000 \\
Independent variable to dependent variable (Path c', control mediating variables ) & 0.104 & 0.038 & 2.758 & 0.006 \\
Results of bootstrap & Lower & Upper \\
\end{tabular}

Note: $N=366$. Leader group prototypicality is independent variable, group commitment is mediating variables and employee well-being is dependent variable.

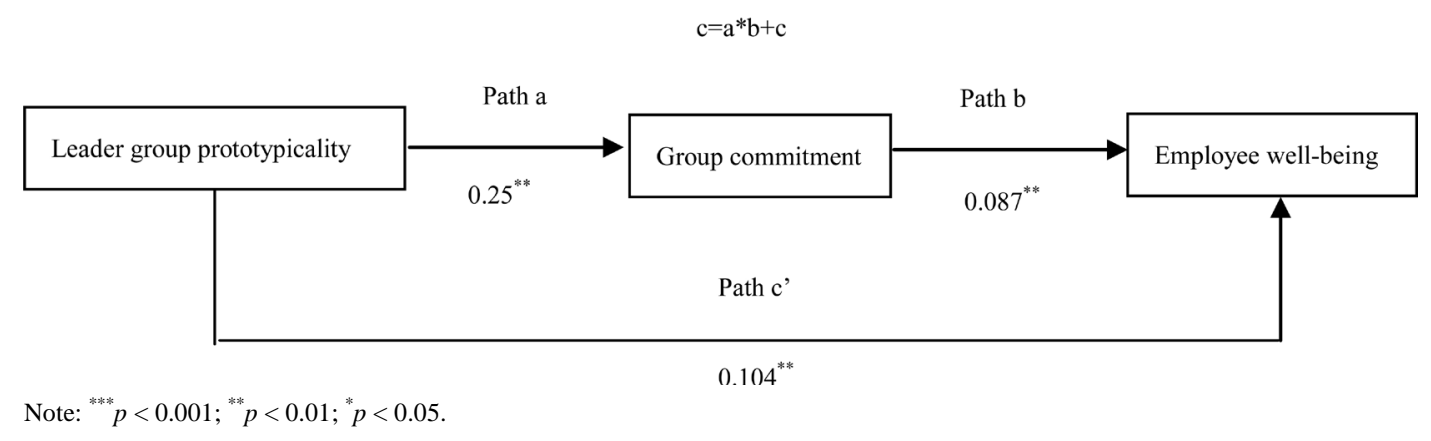

Figure 1. Mediating effects analysis of group commitments.

leader group prototypicality still have a significant effect on employee well-being $(B=0.104, p=0.006)$, and the result of bootstrap is [0.0028, 0.0499] in 95\% confidence intervals without 0 . Thus leader group prototypicality has an indirect, positive relationship, via group commitment, with employee well-being, and Hypothesis 3 was supported.

Regression coefficients, standard errors, and model statistics are summarized, while the model's path coefficients are presented graphically in Figure 1.

\subsection{Analyses Openness as a Moderator}

We exam moderating effect of openness by means of PROCESS (Preacher \& Hayes, 2008; updated by Hayes, 2013). Hypothesis 4 was supported. The relationship between leader group prototypicality, group commitments and openness is shown in Figure 2. Under low level of openness ( $-1 \mathrm{SD})$, leader group prototypicality can be a significant predictor of group commitments (simple slope $=0.20, p<0.05)$, while under high level $(+1 \mathrm{SD}$ ), leader group prototypicality can predict group commitments stronger (simple slope $=0.46, p<0.05$ ), Hypothesis 4 was supported.

\section{Discussion and Conclusion}

Generally, in this study the results show that leader group prototypicality has a significant effect on employee well-being through group commitment. Specifically, when employee has a high level of openness, the impaction of leader group prototypicality to group commitment will become stronger. This study validated previous research point of view, there is a positive effect in promoting leader group prototypicality and employee well-being (Cicero, Pierro, \& Knippenberg, 2007; Cicero et al., 2008). That's mean that if the team leader' character can represent his team, or opinions and decisions reflect most of the views of employees, employee well-being will get higher. Based on social identity theory, employees with high organizational identity often have a higher level of motivation, and they are driven by internal motivation in work, therefore they may enjoy the work. On the other hand, leader with high level of group prototypicality can make employee admit what they say and decision making (Wegge et al., 2008). When an employee admits team leader, they more likely have a high organizational identity (Gregory et al., 2005). Due to the positive role of identity, employees tend to have 


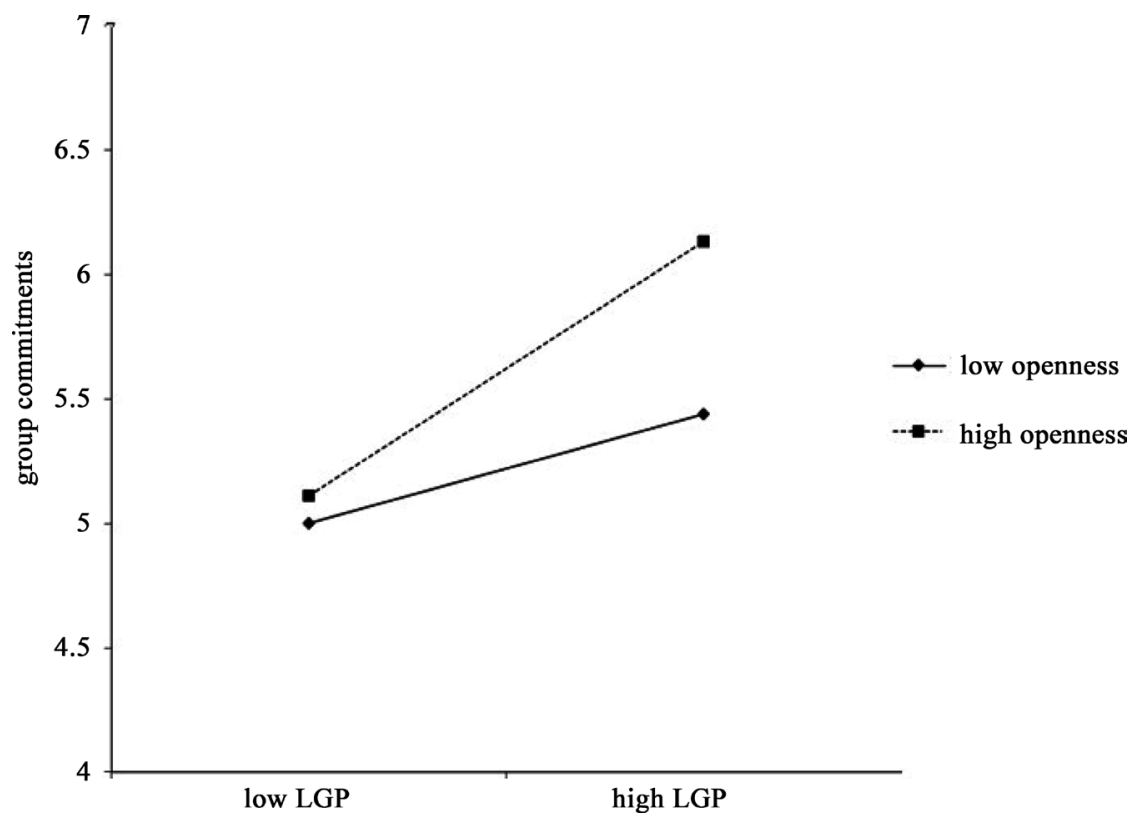

Figure 2. The moderating effect of openness.

group commitment in emotional and cognition, and the high group commitment will improve the well-being of employees.

\subsection{Managerial Implications}

Our findings in this study suggest some managerial implications for organizational in China. According to our results, leader group prototypicality can improve employee's group commitment and employee well-being. Therefore, we can conduct some employee training to improve their group prototypicality of leader, adjust their beliefs, characteristics and perspectives. In this study, we find when openness is stronger, the influence of leader group prototypicality to group commitment can be stronger, too. Thus, it is to make employee conduct an open atmosphere, and make them feel that they can be accept by company.

\subsection{Limitations and Directions for Future Research}

Although our study is helpful to enrich related theories and guide the practice of organizational change in China, there are still several limitations. The first limitation to the current study may provide ideas for future researchers, who can study further to prove the dimensional structure of leader group prototypicality, explore different effects which leader group prototypicality can be influenced. Second, our study mainly focuses on individual perception of leader group prototypicality. However, when individuals are in group or group without obvious boundary, whether the contact within group and the relationship between group members would have impact on the perception of employee well-being. Particularly, when individuals are in different groups, whether there exist different perceptions of leader group prototypicality, those are several directions for future research. Third, because we mainly conduct our survey in city of Guangdong province, the generalizability of the results may be limited. Therefore, this type of survey should be repeated with participants from several areas in China even other countries.

\section{References}

Bakker, A., \& Demerouti, E. (2007). The Job Demands-Resources Model: State of the Art. Journal of Managerial Psychology, 22, 309-328. http://dx.doi.org/10.1108/02683940710733115

Cicero, L., Pierro, A., \& van Knippenberg, D. (2007). Leader Group Prototypicality and Job Satisfaction: The Moderating Role of Job Stress and Team Identification. Group Dynamics, 11, 165-175. http://dx.doi.org/10.1037/1089-2699.11.3.165

Choi, D., Oh, I., \& Colbert, A. (2015). Understanding Organizational Commitment: A Meta-Analytic Examination of the 
Roles of the Five-Factor Model of Personality and Culture. Journal of Applied Psychology, 100, 1542-1567. http://dx.doi.org/10.1037/apl0000014

De Cremer, D., Dijke, M., \& Mayer, D. (2010). Cooperating When "You” and “I” Are Treated Fairly: The Moderating Role of Leader Prototypicality. The Journal of Applied Psychology, 95, 1121-1133. http://dx.doi.org/10.1037/a0020419

Giessner, S., \& van Knippenberg, D. (2008). "License to Fail”: Goal Definition, Leader Group Prototypicality, and Perceptions of Leadership Effectiveness after Leader Failure. Organizational Behavior and Human Decision Processes, 105, 1435. http://dx.doi.org/10.1016/j.obhdp.2007.04.002

Hayes, A. F. (2013). Introduction to Mediation, Moderation, and Conditional Process Analysis: A Regression-Based Approach (pp. 34-40). London \& New York: Guilford Press.

Hogg, M. (2000). Subjective Uncertainty Reduction through Self-Categorization: A Motivational Theory of Social Identity Processes. European Review of Social Psychology, 11, 223-255. http://dx.doi.org/10.1080/14792772043000040

Hogg, M. (2007). Uncertainty-Identity Theory. In M. P. Zanna (Ed.), Advances in Experimental Social Psychology (pp. 39. 69-126). San Diego, CA: Academic Press. http://dx.doi.org/10.1016/s0065-2601(06)39002-8

Kuoppala, J., Lamminpää, A., Liira, J., \& Vainio, H. (2008). Leadership, Job Well-Being, and Health Effects: A Systematic Review and a Meta-Analysis. Journal of Occupational and Environmental Medicine, 50, 904-915. http://dx.doi.org/10.1097/JOM.0b013e31817e918d

Pierro, A., Cicero, L., Bonaiuto, M., van Knippenberg, D., \& Kruglanski, A. (2005). Leader Group Prototypicality and Leadership Effectiveness: The Moderating Role of Need for Cognitive Closure. The Leadership Quarterly, 16, 503-516. http://dx.doi.org/10.1016/j.leaqua.2005.06.002

Ryff, C., \& Dinger, B. (2006). Best News Yet on the Six-Factor Model of Well-Being. Social Science Research, 35, 11031119. http://dx.doi.org/10.1016/j.ssresearch.2006.01.002

Skakon, J., Nielsen, K., Borg, V., \& Guzman, J. (2010). Are Leaders’ Well-Being, Behaviors and Style Associated with the Affective Well-Being of Their Employees? A Systematic Review of Three Decades of Research. Work \& Stress, 24, 107139. http://dx.doi.org/10.1080/02678373.2010.495262

Slemp, G. R., \& Vella-Brodrick, D. A. (2014). Optimising Employee Mental Health: The Relationship between Intrinsic Need Satisfaction, Job Crafting, and Employee Well-Being. Journal of Happiness Studies, 15, 957-977. http://dx.doi.org/10.1007/s10902-013-9458-3

Starbuck, C. (2015). An Investigation of the Relationship between Follower Perceptions of Leader Openness to Experience and Follower Job Satisfaction. Journal of Organizational Culture, Communications \& Conflict, 19, 118-130.

Ullrich, J., Christ, O., \& van Dick, R. (2009). Substitutes for Procedural Fairness: Prototypical Leaders Are Endorsed Whether They Are Fair or Not. The Journal of Applied Psychology, 94, 235-244. http://dx.doi.org/10.1037/a0012936

Zhao, H., Seibert, S., \& Lumpkin, G. (2010). The Relationship of Personality to Entrepreneurial Intentions and Performance: A Meta-Analytic Review. Journal of Management, 36, 81-40. http://dx.doi.org/10.1177/0149206309335187 